\title{
AN EFFICIENT NON-INTRUSIVE REDUCED BASIS APPROACH FOR UNCERTAINTY QUANTIFICATION IN CFD
}

\author{
Dinesh Kumar ${ }^{1}$, Mehrdad Raisee ${ }^{2}$, and Chris Lacor ${ }^{1}$ \\ ${ }^{1}$ Fluid Mechanics and Thermodynamics Research Group, Department of Mechanical Engineering \\ Vrije Universiteit Brussel, Pleinlaan 2, 1050 Brussels, BELGIUM \\ e-mail: $\{$ dkumar, chris.lacor $\} @$ vub.ac.be \\ ${ }^{2}$ School of Mechanical Engineering, College of Engineering \\ University of Tehran, P.O.Box:11155-4563, Tehran, IRAN \\ e-mail: mraisee@ut.ac.ir
}

Keywords: Uncertainty Quantification, Polynomial Chaos, Proper Orthogonal Decomposition, Regression, Sampling.

\begin{abstract}
To handle large number of uncertainties efficiently, a proper orthogonal decomposition (POD) based uncertainty quantification (UQ) scheme is developed and validated in this work. The approach is developed within a non-intrusive polynomial chaos (PC) context based on regression. The main idea is to extract the optimal orthogonal basis via inexpensive calculations on a coarse mesh and then use them for the fine scale analysis. To demonstrate the application and validity of the developed reduced basis model, it is applied to two CFD type applications: (1) flow over a 2D RAE2822 airfoil and (2) the NASA rotor 37, an transonic axial flow compressor. In both test cases, geometrical uncertainties are considered. The results are compared with those of the standard polynomial chaos method. The numerical experiments illustrate that the developed non-intrusive model reduction scheme for $U Q$ has similar accuracy as the classical PC approach at a substantially reduced computational cost. It is also found that the memory requirement of the reduced-order model is much lower than that of the standard polynomial chaos method.
\end{abstract}




\section{INTRODUCTION}

In the design of aeronautical systems, computational models are used routinely. Almost all computational models of a real world application contain uncertainties in physical properties, model parameters, initial conditions, boundary conditions and geometry, which in turn, make the solution uncertain or stochastic. Among several non-intrusive stochastic methods, polynomial chaos method has been implemented by various researchers for a wide range of problems $[1,2,3,4,5]$. Non-intrusive polynomials chaos (NIPC) methods are sampling based methods and the number of simulation calls increases exponentially with increasing number of uncertain parameters. This effect is referred to as the curse of dimensionality.

For uncertainty management and optimization of engineering products, it is necessary to include all important sources of uncertainty in the simulations. This in turn can dramatically increase the computational cost and memory requirement which is undesirable for design purposes. The main challenge industrial applications of uncertainty quantification (UQ) are facing, is the curse of dimensionality as a result of a large number of uncertainties. From an industrial point of view, developing efficient reduced order models to reduce the computational cost and data storage is of great interest for the prediction of complex flows involving a large number of uncertain parameters. In last few years, several model reduction techniques have been proposed to tackle curse of dimensionality $[6,7]$. Reduced basis techniques provide low dimensional approximation of high dimensional outputs. Proper orthogonal decomposition is a popular technique for finding reduced basis. Doostan et al. [7] proposed an intrusive model reduction technique and applied it to a $2 \mathrm{D}$ stochastic solid mechanics problem. Here the approach is extended to non-intrusive polynomial chaos and is applied to $2 \mathrm{D}$ and $3 \mathrm{D}$ industrial applications.

In this work, a regression-based non-intrusive reduced basis technique is developed. To validate the developed reduced basis model, it is applied to two CFD type applications. Firstly, the transonic flow over a 2D RAE2822 airfoil. Secondly, a 3D and more industrial type of application namely the NASA rotor 37, an transonic axial flow compressor. In Section 2, the classical regression-based NIPC scheme is described. In Section 3, the model reduction methodology is presented. Finally in Section 4, the stochastic results are presented and discussed.

\section{NON-INTRUSIVE POLYNOMIAL CHAOS METHOD}

To propagate and quantify uncertainties in a complex problem non-intrusive methods can be applied without any modifications in the deterministic codes. The non-intrusive PC method proceeds as follows:

1. All uncertain parameters and outputs are decomposed in a basis of complete orthogonal polynomials. For example if $k$ is an uncertain parameter of a known probability distribution and $u$ is the output stochastic solution in a mathematical model, the uncertain input parameter $k(x ; \xi)=\sum_{i=0}^{P} k_{i}(x) \psi_{i}(\xi)$ and the output solution $u(x ; \xi)=\sum_{i=0}^{P} u_{i}(x) \psi_{i}(\xi)$ can be written as polynomial chaos expansion. Here $k_{i}$ and $u_{i}$ are deterministic coefficients and $\psi$ are orthogonal polynomials which are function of $\xi=\left(\xi_{1}, \xi_{2}, \xi_{3} \ldots . \xi_{n_{s}}\right)$ where $\xi_{j}$ is a random variable. The coefficients $k_{i}$ can be calculated as the probability distribution of $k$ is known. $n_{s}$ is the number of random dimensions. The total number of terms $P+1$ depends on the highest order of polynomial $(p)$ and on the number of random dimensions as: $P+1=\frac{\left(n_{s}+p\right) !}{n_{s} ! p !}$.

2. Based on the sampling strategy of interest, samples of $\xi^{j}=\left(\xi_{1}, \xi_{2}, \xi_{3} \ldots . \xi_{n_{s}}\right)^{j}$ are gener- 
ated.

3. For each sample $\xi^{j}$, one evaluates the output $u^{j}$ using deterministic solver.

4. Using all $\mathrm{N}$ samples, one numerically evaluates the deterministic coefficients $u_{i}$ for all $i \in[0,1 \ldots P]$.

5. From the deterministic PC coefficients, $u_{o}(x)$ is the mean and the variance of the solution can be calculated as:

$$
\sigma^{2}=\sum_{i=1}^{P} u_{i}^{2}(x)<\psi_{i}^{2}(\xi)>
$$

To compute the polynomial coefficients $u_{i}$ from the solution samples $u^{k}$, numerous methods (e.g. collocation, regression, quadrature method) have been proposed and successfully applied by several researchers to fluid mechanics and solid mechanics problems. In the proposed reduced basis approach we consider the regression method.

\subsection{REGRESSION METHOD}

The regression based non-intrusive PC method starts with replacing the uncertain variables with their polynomial expansions given by the output solution. The output solution in terms of $\mathrm{PC}$ expression is written as:

$$
\sum_{i=0}^{P} u_{i}(x) \psi_{i}(\xi)=u(x ; \xi)
$$

$P+1$ random vectors $\left(\xi^{i}=\left\{\xi_{1}, \xi_{2} \ldots . \xi_{n_{s}}\right\}^{i} ; i=1,2,3 \ldots P+1\right)$ are chosen in random space and the deterministic output is evaluated at these points. A linear system of equations can be obtained as:

$$
\left(\begin{array}{cccccc}
\psi_{0}\left(\xi^{1}\right) & \psi_{1}\left(\xi^{1}\right) & . & . & . & \psi_{P}\left(\xi^{1}\right) \\
\psi_{0}\left(\xi^{2}\right) & \psi_{1}\left(\xi^{2}\right) & . & . & . & \psi_{P}\left(\xi^{2}\right) \\
\psi_{0}\left(\xi^{3}\right) & \psi_{1}\left(\xi^{3}\right) & . & . & . & \psi_{P}\left(\xi^{3}\right) \\
\cdot & \cdot & . & \cdot & \cdot & \cdot \\
\cdot & \cdot & . & \cdot & \cdot & \cdot \\
\psi_{0}\left(\xi^{P+1}\right) & \psi_{1}\left(\xi^{P+1}\right) & . & . & \cdot & \psi_{P}\left(\xi^{P+1}\right)
\end{array}\right)\left(\begin{array}{c}
u_{0} \\
u_{1} \\
u_{2} \\
\cdot \\
\cdot \\
u_{P}
\end{array}\right)=\left(\begin{array}{c}
u\left(\xi^{1}\right) \\
u\left(\xi^{2}\right) \\
u\left(\xi^{3}\right) \\
\cdot \\
\cdot \\
u\left(\xi^{P+1}\right)
\end{array}\right)
$$

or

$$
A u=b
$$

If more than $P+1$ samples are chosen, the over-determined system of equations can be solved using the least squares method. Different sampling approaches can be used: random sampling, Latin hypercube, sampling in (sparse) numerical quadrature points, Sobol sampling etc. Consistent with the literature, we found that over-sampling with $2(P+1)$ simulations is sufficient 


\begin{tabular}{|l|l|l|l|l|}
\hline & $n_{s}=2$ & $n_{s}=4$ & $n_{s}=8$ & $n_{s}=12$ \\
\hline$p=1$ & 6 & 10 & 18 & 26 \\
\hline$p=2$ & 12 & 30 & 90 & 182 \\
\hline$p=4$ & 30 & 140 & 990 & 3640 \\
\hline$p=6$ & 56 & 420 & 6006 & 37128 \\
\hline$p=8$ & 90 & 990 & 25740 & 251940 \\
\hline
\end{tabular}

Table 1: Number of deterministic samples $2(P+1)$ in the regression based UQ as a function of the number of random variables $\left(n_{s}\right)$ and $\mathrm{PC}$ order $(p)$

to achieve satisfactory results. In order to validate the developed model with different sampling schemes, here the Sobol quasi-random sequence (for RAE2822) and the sparse numerical quadrature sequence (for rotor 37 ) with over-sampling of $2(P+1)$ simulations is used to generate the sample points.

The computational cost of the non-intrusive polynomial chaos method is dominated by the computation of $u\left(\xi^{k}\right)$ for every sample $k$. The total number of samples $2(P+1)$ increases exponentially with increasing PC order and number of uncertain parameters (see Table 1). This is the so called "curse of dimensionality".

\section{MODEL REDUCTION METHODOLOGY}

In a reduced basis approach the number of stochastic dimensions can be reduced significantly. The optimal basis can be found from a POD of the solution field (also known as Karhunen-Loève expansion). This requires the knowledge of the covariance of the solution in the stochastic space. However, the solution and hence also its covariance are unknown. Assuming that errors in stochastic space are more or less independent of the discretization errors, the covariance can be obtained via inexpensive calculations on a coarse grid. The resulting optimal basis, $\left\{z_{i}\right\}_{i=1}^{m}$ where $m$ is the number of dominating eigenvalues of the POD, is then used on the fine mesh. Since POD is a covariance based method, the covariance matrix on the coarse grid is obtained using a classical polynomial chaos method.

In the classical polynomial chaos expansion, the random output solution $u(x ; \xi)$ can be decomposed into deterministic and stochastic components. The PC representation of the output solution of order $p$ for $n_{s}$ random variable $\xi \equiv\left\{\xi_{i}\right\}_{i=1}^{n_{s}}$ can be written as:

$$
u(x ; \xi)-<u(x)>=\sum_{i=1}^{P} u_{i}(x) \psi_{i}(\xi)
$$

where $\left\langle u(x, \xi)>\right.$ is the mean value of the stochastic solution $u(x ; \xi)$ and is equal to $u_{0}$.

On the fine grid, an optimal PC expansion of the stochastic output field $u(x ; \xi)$ can be expressed as:

$$
u(x ; \xi)-<u(x)>=\sum_{i=1}^{m} \hat{u}_{i}(x) z_{i}(\xi)
$$

The upper limit $m$ in the above equation can be computed by the size of dominating eigenvalues such that $\sum_{i=1}^{m} \nu_{i} / \sum_{i} \nu_{i} \geq \epsilon$ where $\nu_{i}$ is the eigenvalue of covariance kernel and $\epsilon$ is sufficiently close to one. The number of dominating eigenvalues $(m)$ depends on the chosen value of $\epsilon$. A larger value of $\epsilon$ will give a larger number of modes and so a more accurate solution. 
The covariance function $C\left(x_{1} ; x_{2}\right)$ can be computed from the output solution field on the coarse grid as:

$$
C\left(x_{1} ; x_{2}\right)=\sum_{i=1}^{P} u_{i}\left(x_{1}\right) u_{i}\left(x_{2}\right)<\psi_{i}^{2}>
$$

The eigenvalues $\nu_{i}$ and corresponding eigenfunctions $\phi_{i}(x)$ are the solution of the following eigenvalue problem:

$$
C \phi_{i}=\nu_{i} \phi_{i}
$$

The set of optimal basis $\left\{z_{i}\right\}_{i=1}^{m}$ can be written as a linear combination of classical polynomials $\left\{\psi_{i}\right\}_{i=1}^{P}$ using scalar product ${ }^{1}$ as:

$$
z_{i}(\xi)=\left[u(x ; \xi)-<u(x)>, \phi_{i}(x)\right]=\sum_{j=1}^{P} \alpha_{i j} \psi_{j}(\xi)
$$

One now does the polynomial chaos on a fine mesh, where $z_{i}$ are used instead of $\psi_{i}$. Here again we consider a regression based polynomial chaos approach for fine grid analysis. For $1 \leq i \leq m$, the coefficients in expansion (6) are obtained from linear system of equations as:

$$
\left(\begin{array}{cccccc}
z_{0}\left(\xi^{1}\right) & z_{1}\left(\xi^{1}\right) & \cdot & \cdot & \cdot & z_{m}\left(\xi^{1}\right) \\
z_{0}\left(\xi^{2}\right) & z_{1}\left(\xi^{2}\right) & \cdot & \cdot & \cdot & z_{m}\left(\xi^{2}\right) \\
\cdot & \cdot & \cdot & \cdot & \cdot & \cdot \\
\cdot & \cdot & \cdot & \cdot & \cdot & \cdot \\
z_{0}\left(\xi^{m+1}\right) & z_{1}\left(\xi^{m+1}\right) & \cdot & \cdot & \cdot & z_{m}\left(\xi^{m+1}\right)
\end{array}\right)\left(\begin{array}{c}
\hat{u}_{0} \\
\hat{u}_{1} \\
\cdot \\
\cdot \\
\hat{u}_{m}
\end{array}\right)=\left(\begin{array}{c}
u\left(\xi^{1}\right) \\
u\left(\xi^{2}\right) \\
\cdot \\
\cdot \\
u\left(\xi^{m+1}\right)
\end{array}\right)
$$

The over determined system of equations can be solved using least squares method by choosing $2(m+1)$ samples on the fine grid.

\section{STOCHASTIC APPLICATIONS}

To demonstrate the ability of the developed reduced basis model, the approach is applied to a $2 \mathrm{D}$ transonic airfoil RAE2822 and a 3D and more industrial type of application namely the NASA rotor 37. In both test cases, geometrical uncertainties are considered. For the first test case, the surface of the airfoil is assumed to be a random field with given covariance and is modeled using KL expansion. This allow the generation of a large number of uncertainties. For the second test case, the NASA rotor 37, the geometry of the rotor is parameterized using AutoBlade, a parametric modeler of NUMECA. The rotor 37 blade is parameterized into six sections of 2D airfoils. For each airfoil, leading edge radii and trailing edge radii are considered as uncertain, so that in total 12 uncertain parameters define the geometrical uncertainty of the blade.

\subsection{Transonic RAE2822 airfoil}

The RAE2822 was used as an uncertainty quantification test case in $[8,9]$. Due to the development of an unsteadiness triggered by compressibility effects and shock waves, the two dimensional (2D) transonic flow around a RAE2822 airfoil represents a challenging configuration to investigate the performance of the present reduced order model. The geometry of the

\footnotetext{
${ }^{1}$ The scalar product of functions $v$ and $w$ is defined as: $[v, w]=\int_{x} v \cdot w d x$.
} 


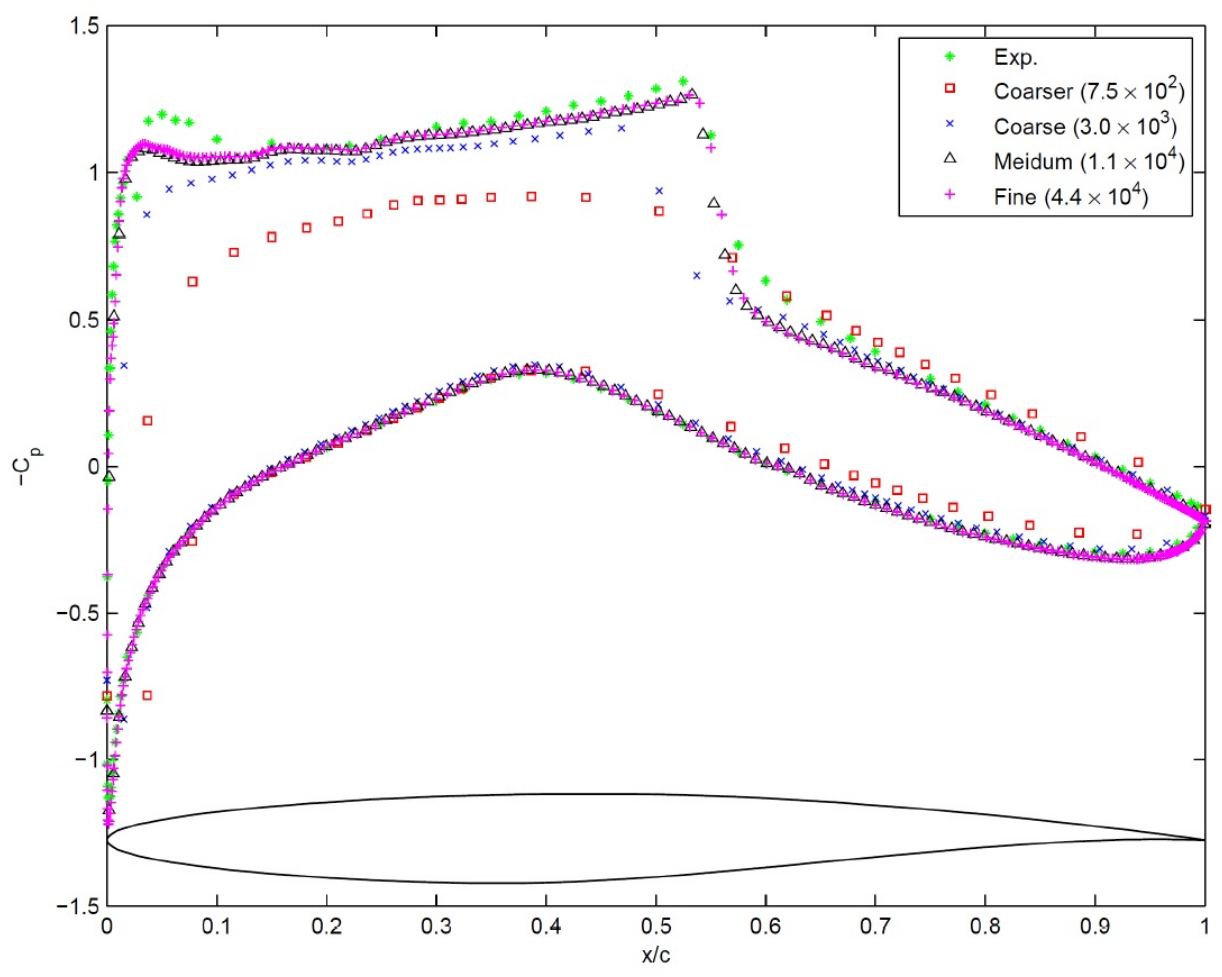

Figure 1: Comparison of the predicted pressure coefficient (deterministic) using different meshes with the experimental data of Cook et al. and Schematic of RAE2822 transonic airfoil

transonic airfoil RAE2822 is described in Cook et al.[10]. The nominal flow conditions correspond to free stream Mach number $M_{\infty}=0.734$, Reynolds number $R e=6.5 \times 10^{5}$ and angle of attack $\alpha=2.79^{\circ}$. For deterministic CFD solutions, Ansys/Fluent is used as a block box solver. A second-order upwind scheme is employed for the approximation of nonlinear convective terms in all transport equations. The Spalart-Allmaras one-equation turbulence model is used for turbulence modeling. The CFD results are validated against experimental data [10] in figure 1. Increasing the number of grid nodes improve the pressure coefficient predictions and the numerical results on a fine mesh with $4.4 \times 10^{4}$ nodes are grid independent.

In order to make this test case as a high dimensional stochastic problem, uncertainty is introduced in the geometry of airfoil. The geometrical roughness, corresponding to the manufacturing tolerances, is modeled as a stochastic process. Here a Gaussian process is assumed with zero mean and a covariance function given by

$$
\operatorname{Cov}\left(s_{i}, s_{j}\right)=\sigma\left(s_{i}\right) \sigma\left(s_{j}\right) e^{-\frac{\left(s_{i}-s_{j}\right)^{2}}{2 b^{2}}}
$$

where $s_{i}$ and $s_{j}$ are positions along the airfoil, $b$ is the correlation length and $\sigma$ is the variance. For the RAE2822 airfoil, $0 \leq s \leq 2.032$. Position $s=0$ corresponds to the trailing edge and increases along the upper surface.

Using Karhunen-Loève expansion, a stochastic process of a given covariance function, can always be approximated by a finite sum of products of deterministic spatial functions and random variables as: 


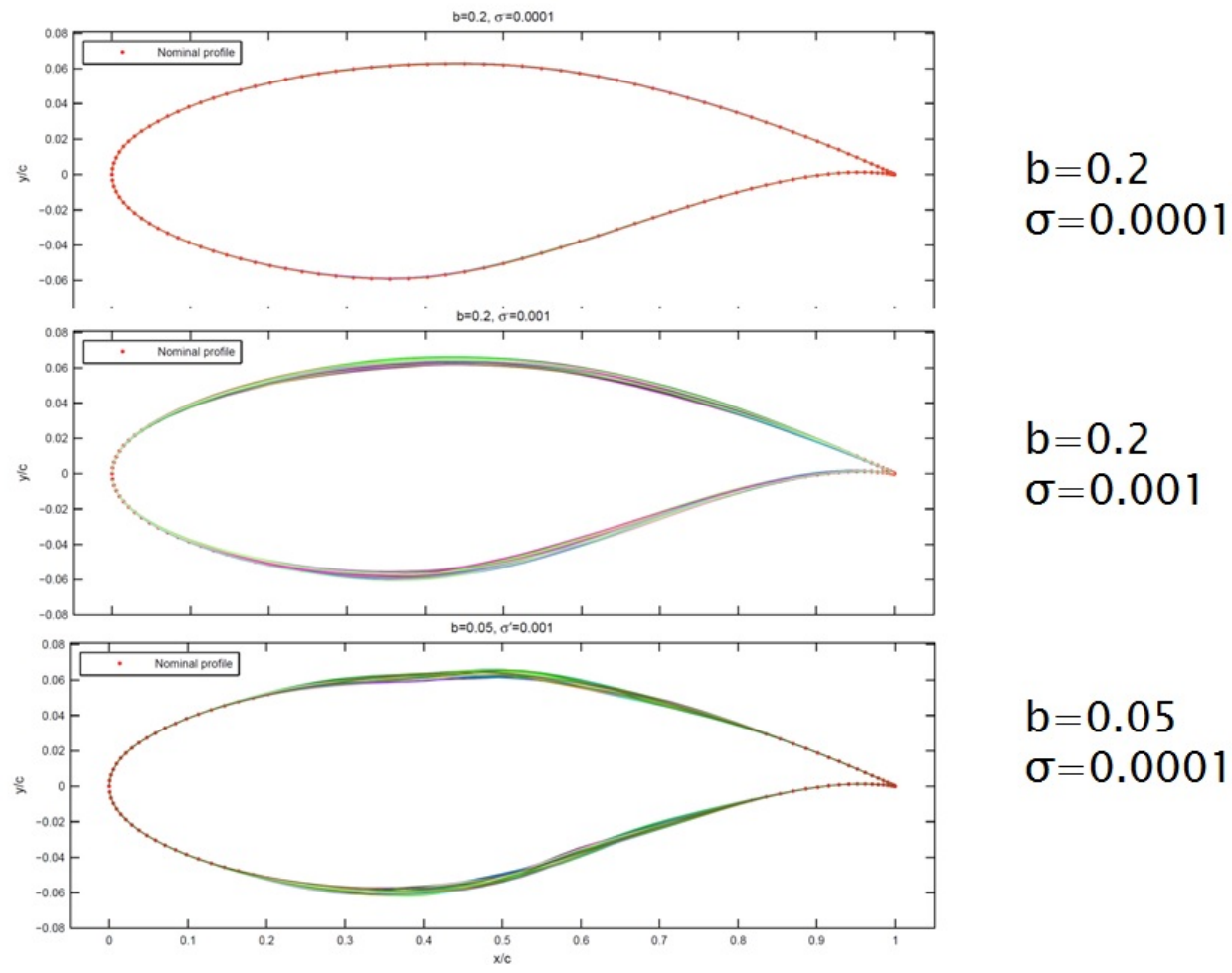

Figure 2: RAE2822 airfoil geometry realizations with different correlation length (b) and variance $(\sigma)$ for Gaussian covariance function.

$$
k(x ; \omega)=k_{0}+\sum_{i=1}^{m} \sqrt{\lambda_{i}} \phi_{i}(x) \xi_{i}(\omega)
$$

where $k_{0}$ is the mean field, $\xi_{i}$ are uncorrelated random variables and $\lambda_{i}$ are the eigenvalues of the covariance kernel.

The geometrical uncertainty at the airfoil surface can then be expressed as:

$$
s(x ; \omega)=s_{0}(x)+\sum_{i=1}^{m} \sqrt{\lambda_{i}} \phi_{i}(x) \xi_{i}(\omega) \cdot \vec{n}
$$

where $\vec{n}(x)$ is a normal vector and $s_{o}(x)$ is the airfoil mean geometry at a point $x$. By generating samples in $\xi_{i}$, the airfoil geometry realizations will be generated. We assume that the random variables are uniformly distributed over the stochastic space $[-1,1]^{n_{s}}$ where $n_{s}$ is the number of random variables taken into the consideration. Ten uniformly distributed independent random variables are considered to create the airfoil realizations. The RAE2822 airfoil geometry realizations with correlation length $(b)$ and variance $(\sigma)$ are shown in figure 2 for 10 input uncertain parameters $\left(n_{s}=10\right)$. A case with correlation length $b=0.05$ and variance $\sigma=0.0001$ is considered for analysis.

In figures 3, 4 and 5, reduced order results for the turbulent flow around the transonic RAE2822 airfoil with geometrical uncertainty are shown and discussed. The reduced-order model results for the mean and standard deviation are compared with those obtained with the 

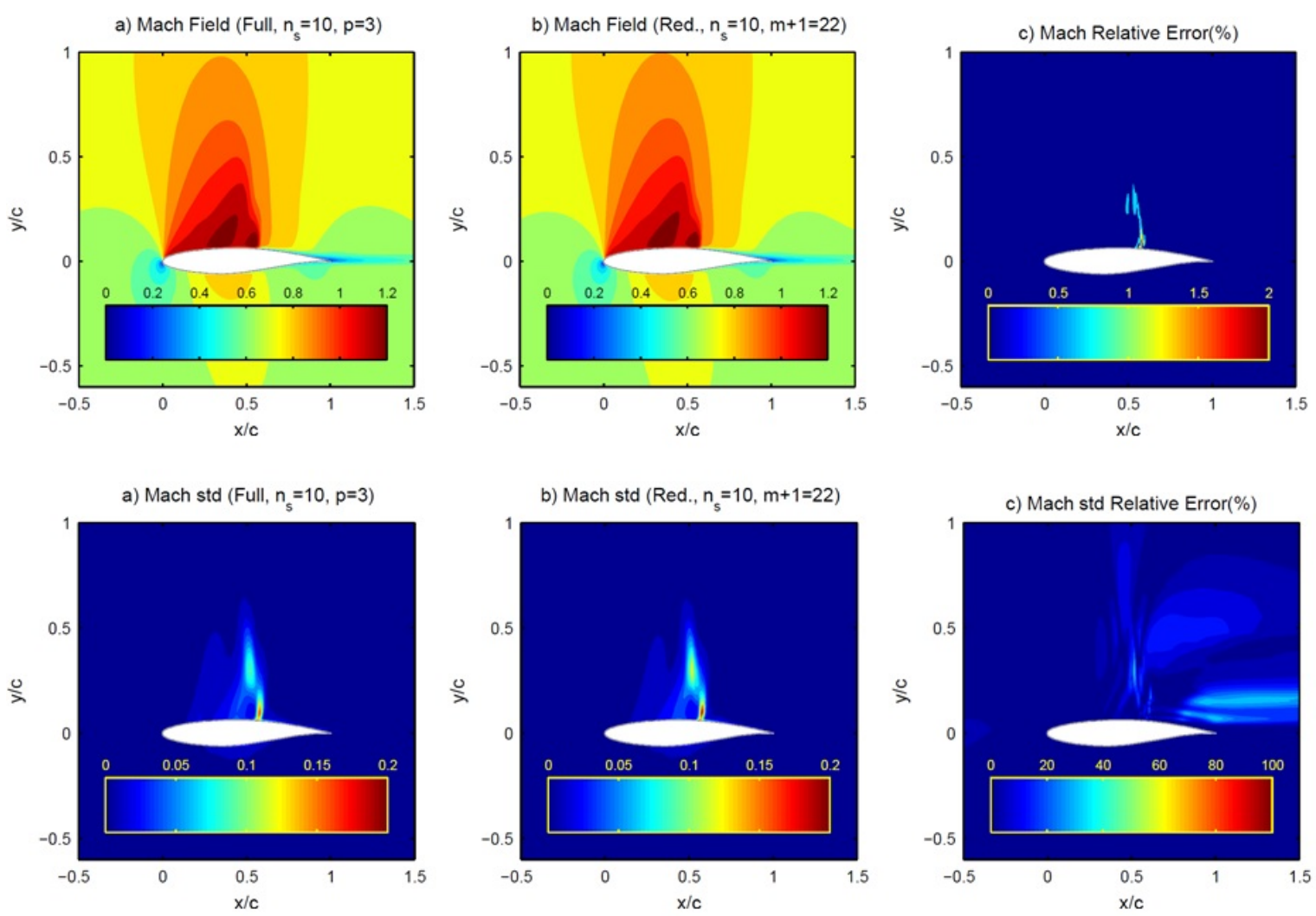

Figure 3: Comparison of Mach field: mean and standard deviation using classical PC method (first column), mean and standard deviation using model reduction (second column) and relative error (third column).

full PC. Third order PC approximation is used as full PC method as well as for the coarse grid analysis of the model reduction technique. The coarse scale analysis was performed on a C-type mesh with $3.0 \times 10^{3}$ nodes. Similarly the fine scale analysis was performed on a fine C-type mesh with $4.4 \times 10^{4}$ grid nods. Note that the ratio of the number of fine mesh nodes to the number of coarse mesh nodes is almost 14 . In this analysis, the covariance matrix is built using all primitive variables $(\rho, \rho u, \rho v, \rho E)$.

By looking at the Mach field and pressure field (figure 3 and 4) the results of the reduced model are in acceptable agreement with the results of the full model. Concerning pressure coefficient $\left(C_{p}\right)$ predictions shown in figure 5 , it is observed that both set of computations give more or less similar results. The largest discrepancy occurs in the shock region. Although high local error is visible (specially in the variance distribution), the results of the reduced model are in acceptable agreement with the results of the full model. The average errors in the mean $C_{p}$ and its variance are less than $0.2 \%$ and $5.0 \%$ respectively.

The size of the reduced basis is 22 (including the zeroth order), requiring 44 deterministic CFD calculations on the fine scale analysis which is cheaper than the cost of 572 fine mesh CFD calculations when a full PC with $n_{s}=10$ and $p=3$ is used. If ' $t$ ' is the time for computing one deterministic solution on the fine grid, the classical PC method will take $572 t$ for 572 samples on the fine grid. Using the reduced basis approach, 572 samples on the coarse grid (14 times coarser) will take $41 t(\approx 572 t / 14)$ and 44 samples on the fine grid will take $44 t$. The total computation time for sampling in reduced basis approach will be approx $85 t$, that is making reduced basis approach almost 6-7 times more efficient compared to the classical PC method. 

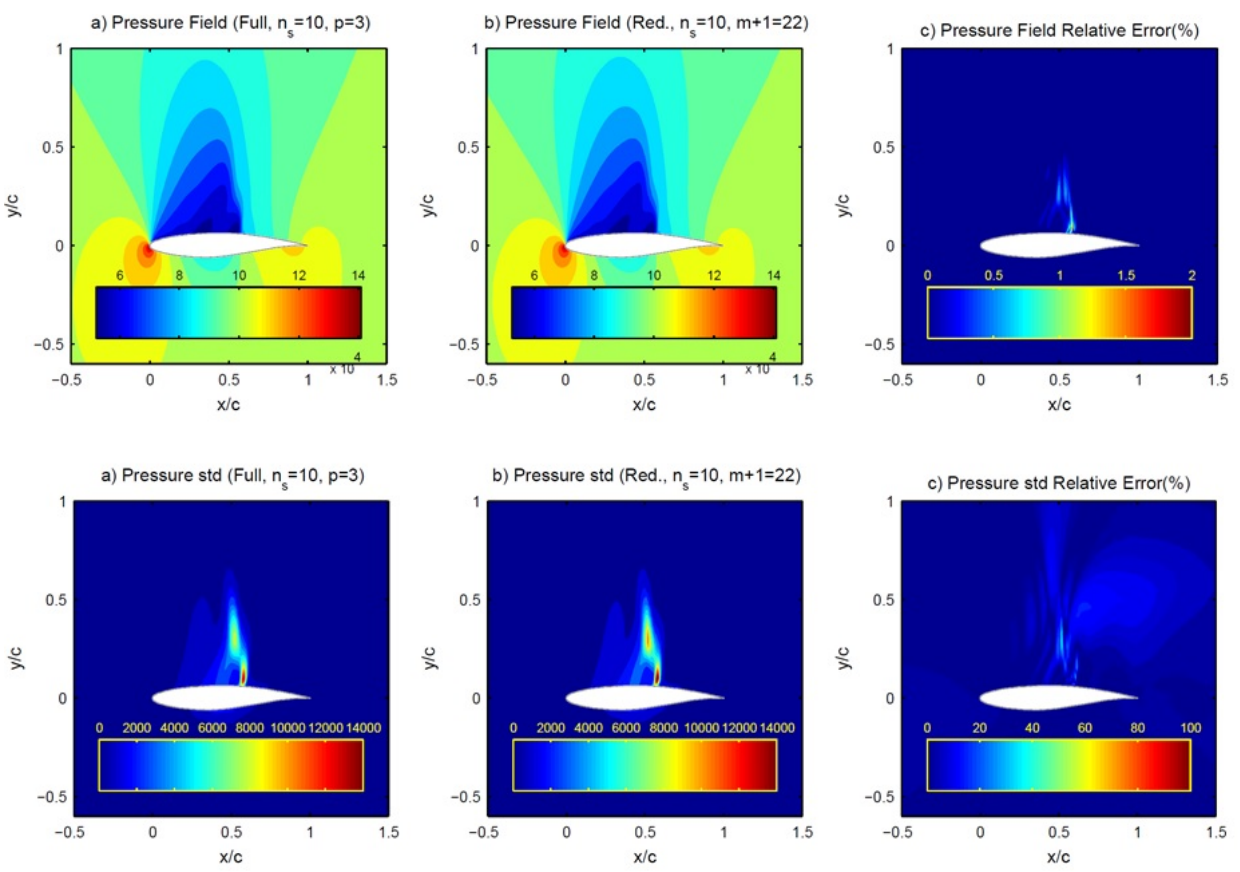

Figure 4: Comparison of pressure field: mean and standard deviation using classical PC method (first column), mean and standard deviation using model reduction (second column) and relative error (third column).

\subsection{Rotor37 axial flow compressor}

The NASA rotor 37, an isolated axial flow compressor was originally designed and experimentally tested by Reid and Moore [11] as part of a NASA research program. This test case was initially included in a wider research program that cover a range of design parameters typically of aircraft turbine engine and it is a commonly used test case for turbomachinery. The rotor 37 is an isolated transonic axial compressor rotor with 36 blades $[12,13]$. Compressor rotors are components of a gas turbine that are highly sensitive to operational and geometrical uncertainties. Efficient uncertainty analysis by considering most of the important uncertain parameters is, therefore, of great importance to assure robustness of the design. Recently, uncertainty quantification is applied to the rotor 37 in order to study the effect of uncertain static outlet pressure [14] and total inlet pressure [15] individually. For the validation of the developed model reduction approach in a complex 3D test case, here we intend to introduce uncertainties in the geometry of the rotor 37 blade to model geometrical roughness.

For deterministic simulations of the rotor 37, FINE/Turbo of NUMECA International is used as a black box solver. The deterministic computation of the rotor 37 consists of one blade with periodic boundary conditions. The flow through NASA rotor 37 is simulated by the Reynolds averaged Navier-Stokes equations in combination with the Spalart-Allmaras turbulence model. The space derivatives are discretised using a central scheme with explicit four stage RungeKutta method. The grid that is used, contains approximately 766,000 cells with proper clustering near solid boundaries, based on the experience of Tartinville and Hirsch [16]. The rotational speed of the rotor is $17188 \mathrm{rpm}$. In the computational model, the shroud is kept fixed while the hub and blade are the moving parts. Inlet boundary conditions for total temperature and total pressure are taken from experimental data [11]. All the simulations in this work are conducted at constant outlet static pressure of $114074 \mathrm{~Pa}$.

The computational model of the compressor rotor 37 is shown in figure 6. A single blade 

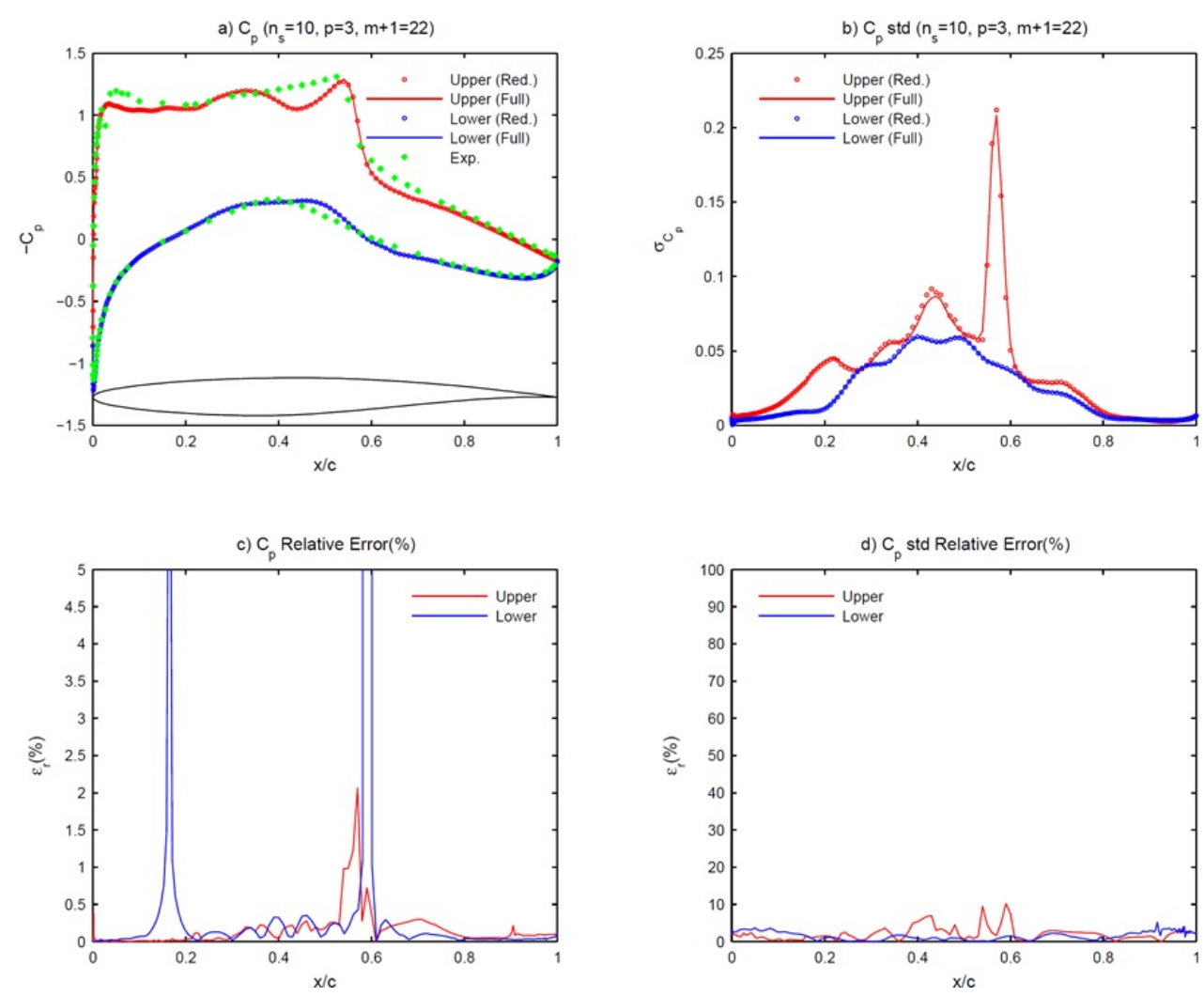

Figure 5: Comparison of pressure coefficients obtained using reduced model and classical PC: mean and standard deviation of pressure coefficient (first row), relative error (\%) in mean and standard deviation of pressure coefficient (second row).

with hub and shroud as well as grid on it is also depicted in the figure. The static pressure on the hub, shroud, $25 \%, 50 \%$ and $75 \%$ span of the blade is shown in figure 7 as a deterministic solution. The pressure distribution around the blade at mid span height is also shown in figure 7.

For uncertainty quantification and validation of developed reduced basis approach, the geometry of the rotor blade is parameterized into six sections of 2D airfoils using AutoBlade, a parametric modeler of NUMECA. For each airfoil, leading edge radii and trailing edge radii (resulting from manufacturing tolerances) are considered as uncertain. Symmetric beta distribution $(\alpha=\beta=4)$ with a minimum as $90 \%$ of most likely values and a maximum as $110 \%$ of most likely values is assumed for all twelve uncertain parameters. Ten geometry realizations of the rotor 37 blade at mid span height are shown in figure 8 . The coarse grid analysis for reduced basis approach is performed with $1.04 \times 10^{5}$ grids and for the fine scale analysis $7.66 \times 10^{5}$ grid nodes are used, that is making fine grid to coarse grid ratio as almost 7.5. Regression based classical polynomial chaos method with second order PC approximation and twelve uncertain parameters requires in total 182 CFD simulations on the fine grid (see Table 1). Due to the large number of CFD simulations and complexity of the present test case, the developed reduced basis approach is first validated for first order classical PC method. Furthermore, with second order PC approximation, the reduced basis approach is used to enhance the accuracy of the statistical quantities. In this analysis, the covariance matrix is constructed using static pressure field only.

For the validation of the model reduction approach and for better visualization, pressure distribution around the blade at mid span height is compared with classical polynomial chaos method (figure 9) for first order PC approximation. In figure 9, the mean (left) and standard 

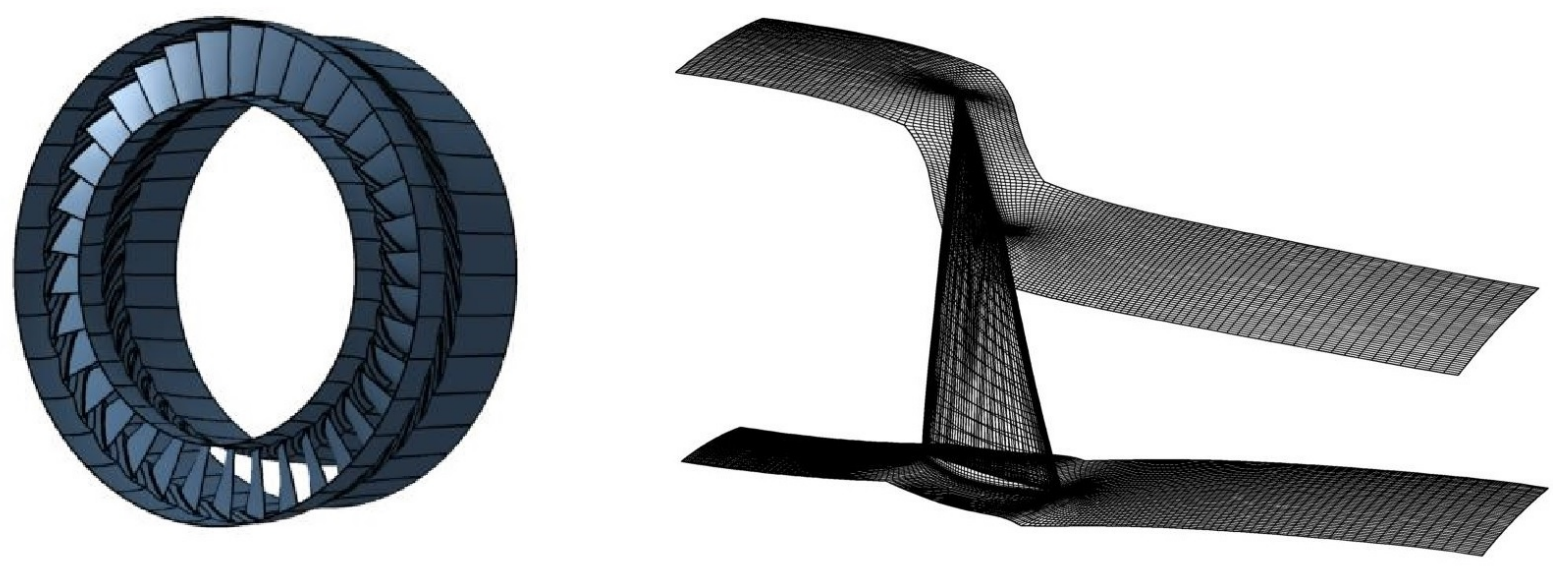

Figure 6: NASA Rotor 37, computation model (left) and single blade with mesh (right)
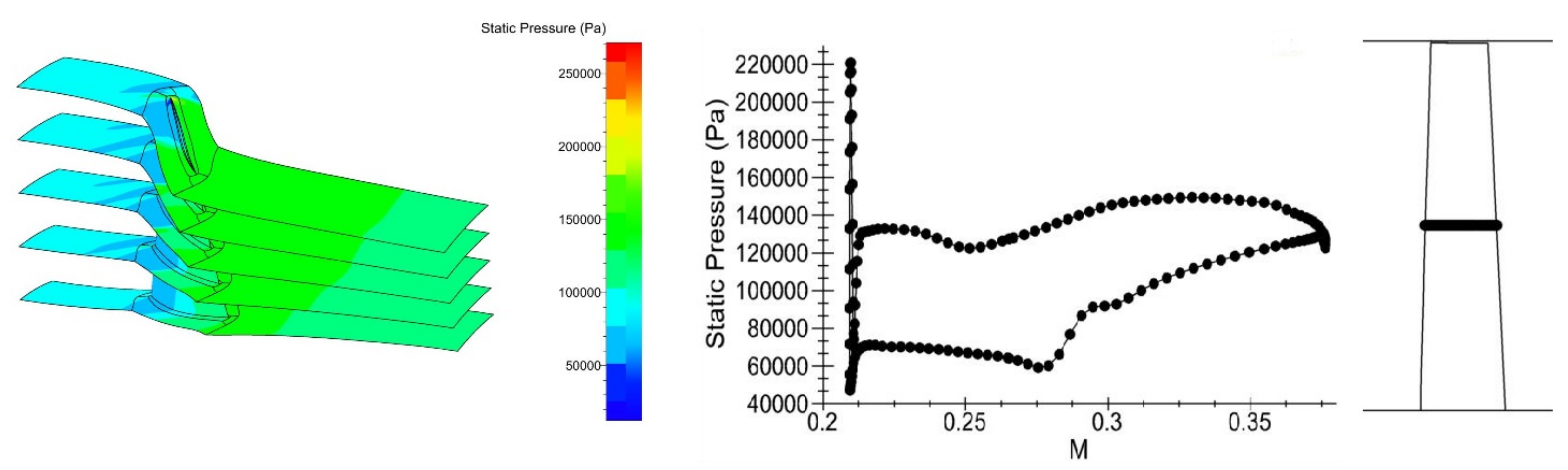

Figure 7: Deterministic solution: static pressure distribution on the blade surface (hub, shroud, $25 \%, 50 \%$ and $75 \%$ span of the blade (left) and pressure distribution at mid span height of the blade (right)

deviation (right) of the static pressure at mid span height of the rotor blade from the reduced basis approach and classical polynomial chaos are shown. As it can be observed from figure 9, the reduced basis and classical PC methods produce similar results.

In figure 10, the relative $\%$ differences in the mean (left) and standard deviation (right) from the reduced basis and classical PC approach is also shown. The reduced basis approach captures the mean values accurately with negligible differences in the standard deviation (less than $5 \%$ ). The differences can be reduced by considering a better sampling scheme as in the least square (regression) methods the selection of the sampling points has a significant impact on the solution. By considering all primitive variables in computation of covariance may also improve the results.

For 12 random variables and a second order PC approximation with the regression method, 182 CFD calculations are needed. The size of the reduced basis $(m+1)$ is 7 (including the zeroth order for $\epsilon=0.999$ ) requiring only 14 CFD calculations on the fine grid. The normalized eigenvalues from the coarse grid analysis (for PC order 1 and 2) are shown in figure 11. From this figure it can be observed that the first six eigenvalues $(m=6)$ are sufficient to capture the statistical results accurately. The mean (left) and standard deviation (right) of the static pressure at mid span height of the rotor blade from the reduced basis approach with enhanced accuracy (PC order 2) are shown in figure 12. If ' $t$ ' is the time for one CFD computation, classical PC 


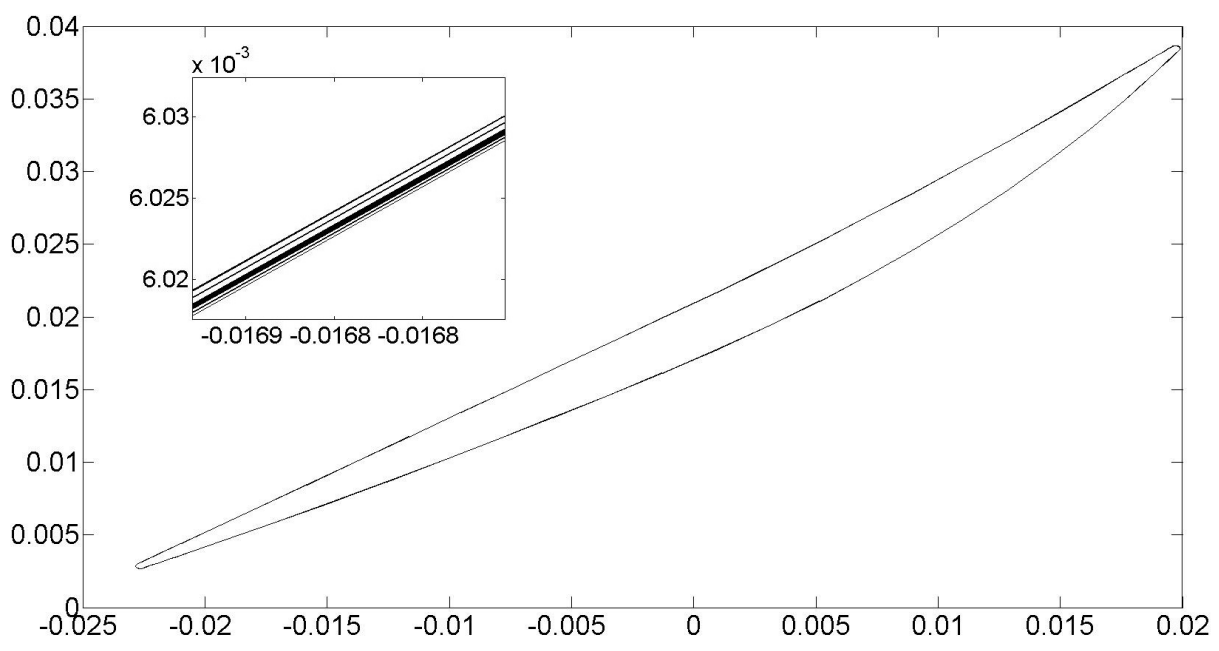

Figure 8: Geometry realizations at mid span height of the rotor blade with uncertain leading and trailing edge radii

method will take $182 t$ for 182 samples on the fine grid. Using reduced basis approach, 182 samples on the coarse grid (almost 7.5 times coarser) takes $24 t(=182 t / 7.5)$ and 14 samples on the fine grid take 14t. Total computation time for the reduced basis approach will be $38 t$, that is making the reduced basis approach almost 5 times more efficient than the classical PC. As expected, the results of the proposed reduced model are in acceptable agreement with the results of classical PC method in 3D CFD computation as well.

\section{SUMMARY AND CONCLUSIONS}

In this paper, a non-intrusive model reduction technique for PC expansion is presented and discussed. The reduced order model is applied to a two-dimensional (RAE2822 airfoil) and a three-dimensional (NASA rotor37) industrial applications with geometrical uncertainties. Distributions of the mean and standard deviation obtained from the reduced order model are compared with those of full order model. The numerical results show that the developed reduced order model is able to produce acceptable results for mean and variance fields. Computation time of the reduced order model is found to be much lower than that of the full order model. From these challenging test cases it can be seen that proposed model reduces computational cost dramatically with very good accuracy in the statistical results.

\section{ACKNOWLEDGMENT}

The authors wish to thank Vrije Universiteit Brussel (VUB) for providing financial support of this work. This work was partly done in the UMRIDA project which is supported by the European Commission under contract No. ACP3-GA-2013-605036.

\section{REFERENCES}

[1] R. Ghanem and P. Spanos, Stochastic Finite Elements: A Spectral Approach. SpringerVerlag, New York, 1991.

[2] S. Hosder, R. W. Walters and R. Perez, A non-intrusive polynomial chaos method for uncertainty propagation in CFD Simulations, 44th AIAA Aerospace Sciences Meeting and 

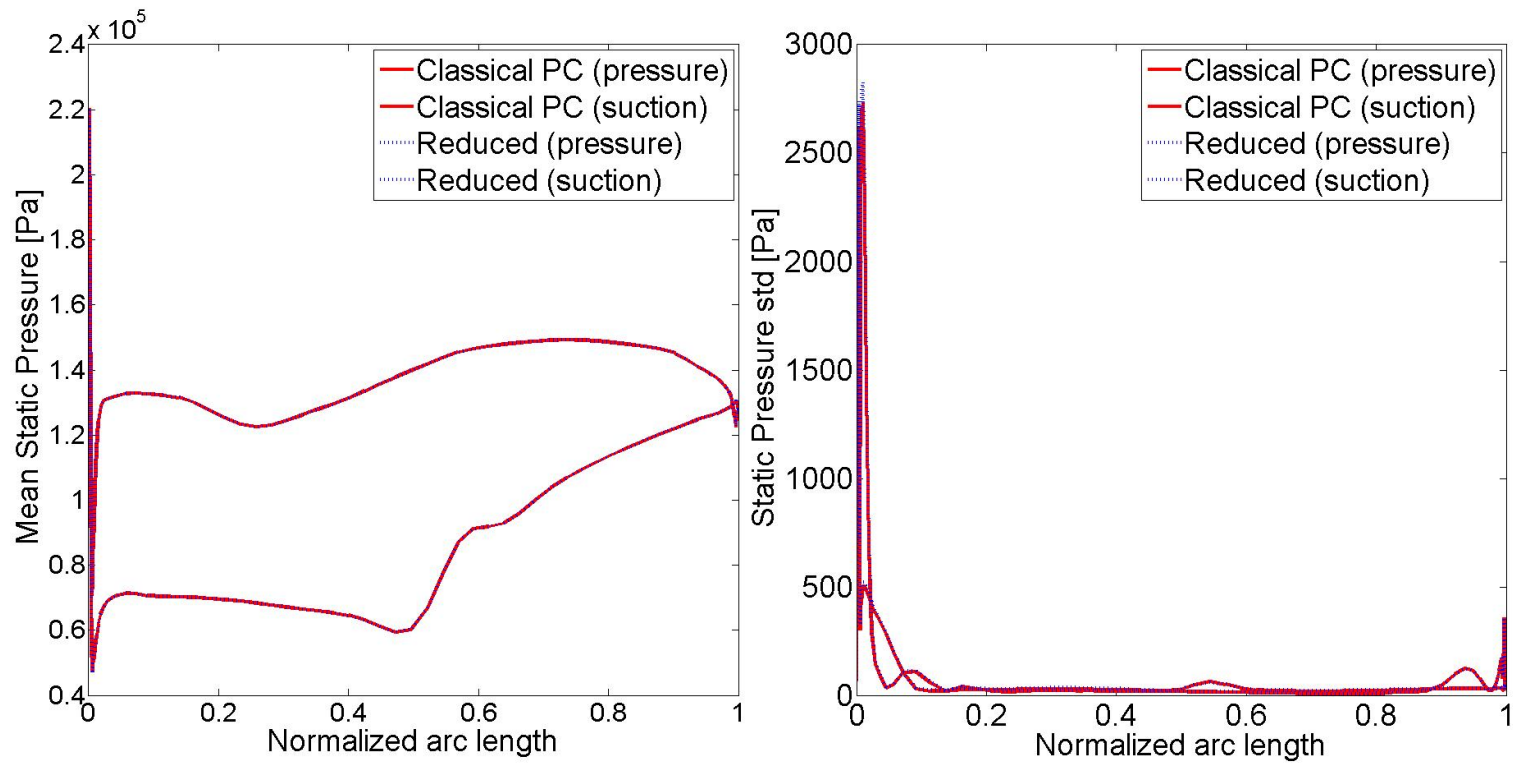

Figure 9: Comparison of the reduced model and classical polynomial chaos: Pressure distribution around the rotor blade: mean (left) and std deviation (right.)
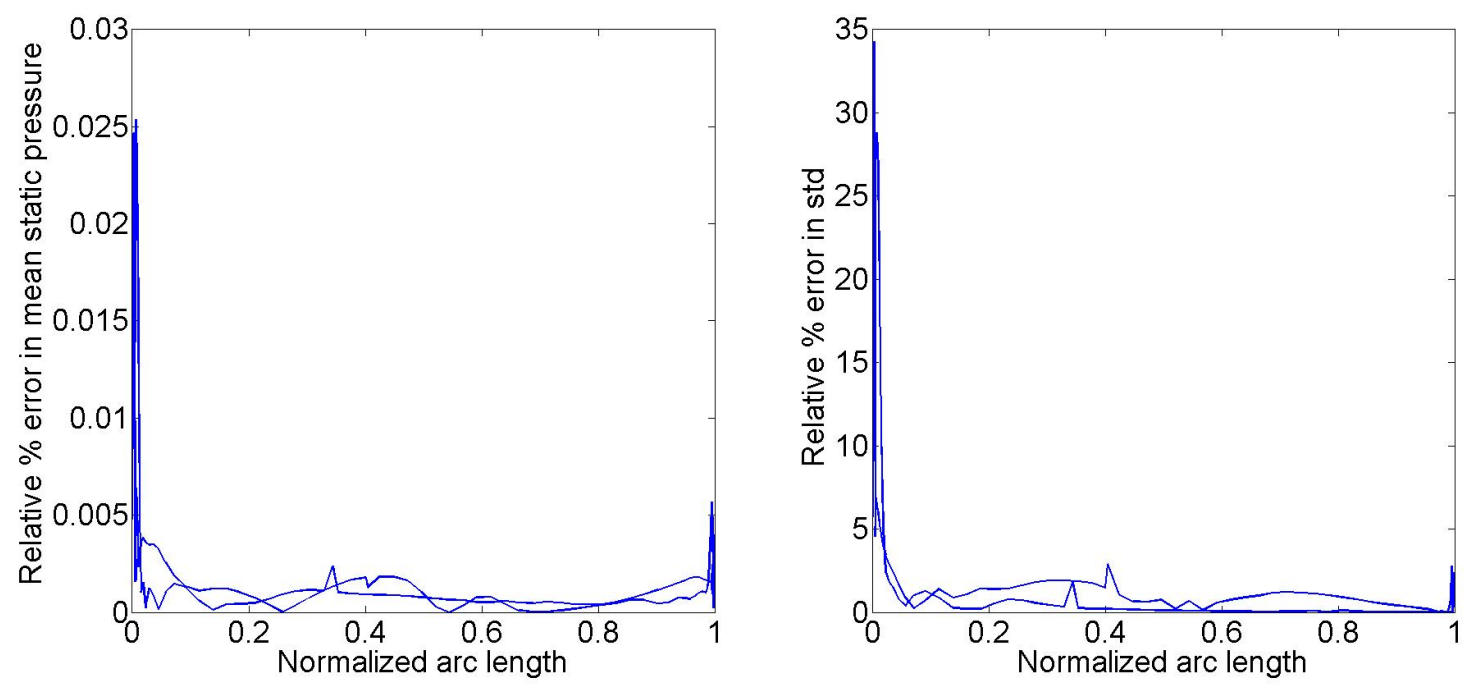

Figure 10: Relative error (\%) in mean and std deviation of the pressure distribution around the rotor blade using the reduced model and classical PC (for PC order 1). 


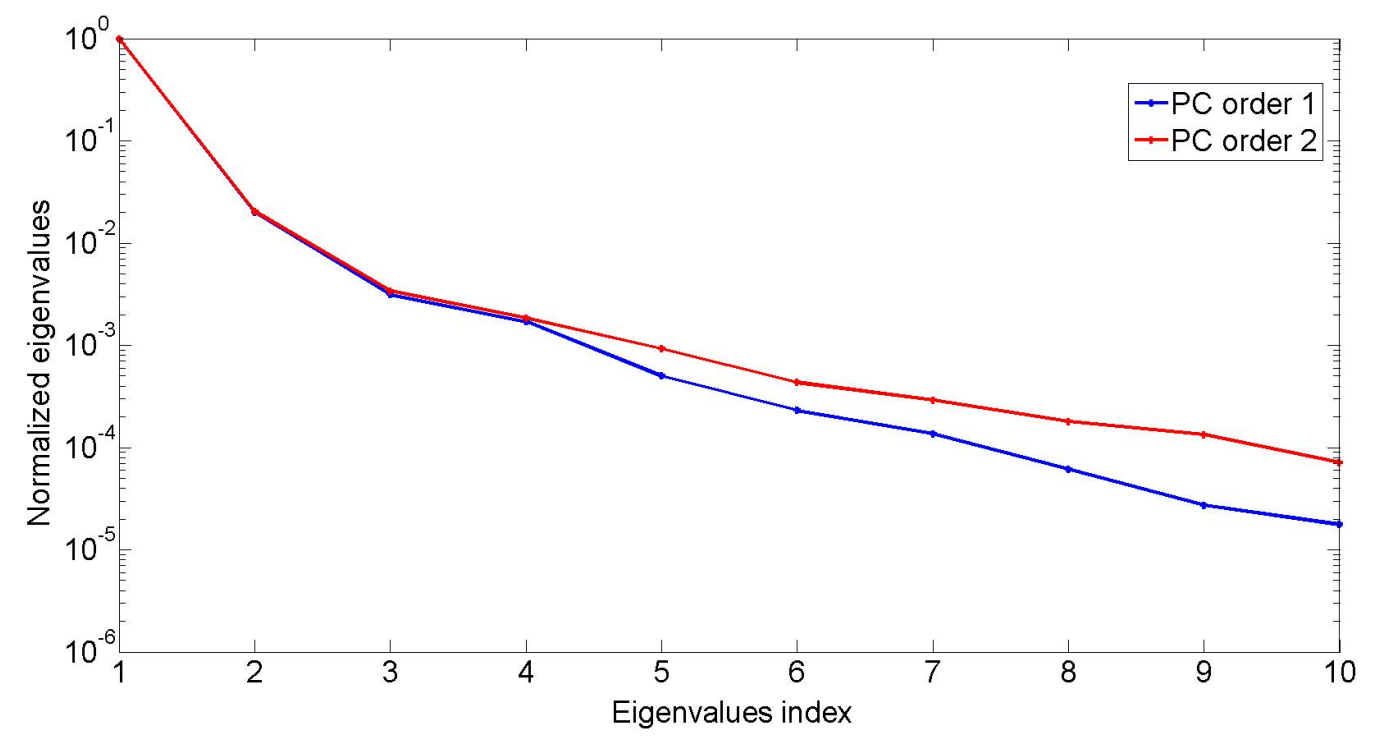

Figure 11: Normalized eigenvalues from the coarse grid analysis for PC order 1 and 2
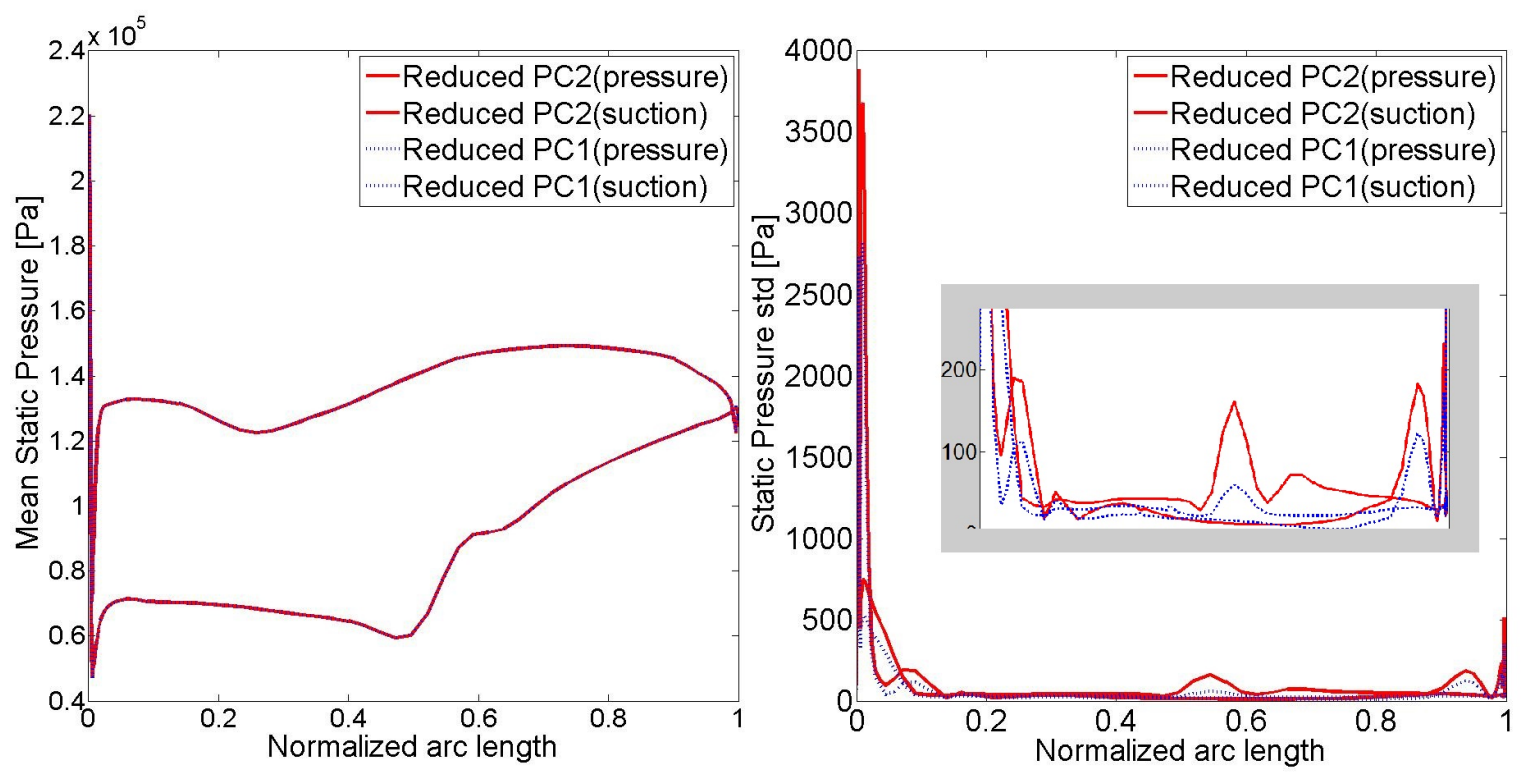

Figure 12: Mean and std deviation of the pressure distribution around the rotor blade using reduced model for PC order 2. 
Exhibit, Reno, Nevada,January 9 - 122006

[3] D. Xiu and G. E. Karniadakis, The wiener-askey polynomial chaos for stochastic differential equations. SIAM Journal on Scientific Computing, 24(2):619644, 2002.

[4] I. Babuska, F. Nobile, and R. Tempone, A stochastic collocation method for elliptic partial differential equations with random input data. SIAM Journal on Numerical Analysis, 45(3):10051034, 2007.

[5] C. Dinescu, S. Smirnov, Ch. Hirsch, and C. Lacor, Assessment of intrusive and nonintrusive non-deterministic CFD methodologies based on polynomial chaos expansions. Int. J. of Eng. Systems Modeling and Simulation, 2:87-98, 2010.

[6] A. Nouy, A generalized spectral decomposition technique to solve a class of linear stochastic partial differential equations. Computer Methods in Applied Mechanics and Engineering 2007; 196(45-48):4521-4537.

[7] A. Doostan, R. Ghanem, and J. Red-Horse. Stochastic model reduction for chaos representations. Comp. Meth. in Appl. Mech. and Eng., 196:3951-3966, 2007.

[8] J. A. S. Witteveen, A. Doostan, R. Pecnik and G. Iaccarino, Uncertainty quantification of the transonic flow around the RAE 2822 airfoil. Center for Turbulence Research Annual Research Briefs 2009.

[9] H. Chen, Q. Wang, R. Hu and P. Constantine, Conditional sampling and experiment design for quantifying manufacturing error of transonic airfoil 49th AIAA Aerospace Sciences Meeting including the New Horizons Forum and Aerospace Exposition 4 - 7 January 2011, Orlando, Florida.

[10] P. H. Cook, M. A. McDonald, M. C. P. Firmin, Aerofoil RAE 2822 - Pressure Distributions, and Boundary Layer and Wake Measurements, Experimental Data Base for Computer Program Assessment, AGARD Report AR 138, 1979.

[11] Reid, Lonnie and Moore, Royce, Performance of Single-Stage Axial-Flow Transonic Compressor With Rotor and Stator Aspect Ratios of 1.19 and 1.26, Respectively, and With Design Pressure Ratio of 1.82, NASA Technical Paper 1338, November 1978.

[12] J. Dunham, CFD validation for propulsion system components, AGARD-AR-355, 1998.

[13] M. R. Simoes, B. G. Montojos, N. R. Moura, J. Su, Validation of turbulence models for simulation of axial flow compressor, Proceedings of COBEM, 2009.

[14] G. J. A. Loeven and H. Bijl, The Application of the Probabilistic Collocation Method to a Transonic Axial Flow Compressor, 51 st AIAA/ASME/ASCE/AHS/ASC Structures, Structural Dynamics, and Materials Conference, 12 - 15 April 2010, Orlando, Florida.

[15] X. Wang, C. Hirsch, Z. Liu, S. Kang, and C. Lacor, Uncertainty-based robust aerodynamic optimization of rotor blades. Int. J. Numer. Meth. Eng.,94:111-127, 2013.

[16] B. Tartinville and C. Hirsch, Rotor 37, in FLOMANIA A European Initiative on Flow Physics Modelling Notes Numerical Fluid Mechanics and Multidisciplinary Design, Vol. 94, pp.183202, Springer 2006. 